\title{
Investigation of the Gravitational Potential Dependence of the Fine-Structure Constant Using Atomic Dysprosium
}

\author{
S.J. Ferrell, ${ }^{1}$ A. Cingöz, ${ }^{1}$ A. Lapierre,${ }^{2}$ A.-T. Nguyen,${ }^{3}$ N. Leefer,${ }^{1}$ D. Budker,${ }^{1,4}$ \\ V.V. Flambaum ${ }^{5,6}$, S.K. Lamoreaux, ${ }^{7}$, and J.R. Torgerson ${ }^{3}$ \\ ${ }^{1}$ Department of Physics, University of California at Berkeley, Berkeley, California 94720-7300, USA \\ ${ }^{2}$ TRIUMF National Laboratory, 4004 Wesbrook Mall, Vancouver, British Columbia, V6T 2A3, Canada \\ ${ }^{3}$ Physics Division, Los Alamos National Laboratory, P-23, MS-H803, Los Alamos, New Mexico 87545, USA \\ ${ }^{4}$ Nuclear Science Division, Lawrence Berkeley National Laboratory, Berkeley, California 94720, USA \\ ${ }^{5}$ School of Physics, The University of New South Wales, Sydney NSW 2052, Australia \\ ${ }^{6}$ Institute for Advanced Study, Massey University (Albany Campus), Private Bag 102904, North Shore MSC Auckland, New Zealand \\ 7 Department of Physics, Yale University, New Haven, Connecticut 06520-8120, USA
}

\begin{abstract}
Radio-frequency E1 transitions between nearly degenerate, opposite parity levels of atomic dysprosium were monitored over an eight month period to search for a variation in the fine-structure constant. During this time period, data were taken at different points in the gravitational potential of the Sun. The data are fitted to the variation in the gravitational potential yielding a value of $(-8.7 \pm 6.6) \times 10^{-6}$ for the fit parameter $k_{\alpha}$. This value gives the current best laboratory limit. In addition, our value of $k_{\alpha}$ combined with other experimental constraints is used to extract the first limits on $k_{e}$ and $k_{q}$. These coefficients characterize the variation of $m_{e} / m_{p}$ and $m_{q} / m_{p}$ in a changing gravitational potential, where $m_{e}, m_{p}$, and $m_{q}$ are electron, proton, and quark masses. The results are $k_{e}=(4.9 \pm 3.9) \times 10^{-5}$ and $k_{q}=(6.6 \pm 5.2) \times 10^{-5}$.
\end{abstract}

According to general relativity, values of fundamental constants are independent of space and time as required by the Einstein Equivalence Principle (EEP). Modern theories that aim to unify gravitation with the other forces, however, do not set this restriction on the fundamental constants; so, they may vary either spatially or temporally, which is in conflict with EEP [1].

Various recent studies have reported the results of searches for a temporal variation of the fine-structure constant, $\alpha=e^{2} / \hbar c$. These include the analyses of absorption spectra from quasars [2-5], the analyses of the nuclear products of the natural fission reactor at Oklo, which operated $2 \times 10^{9}$ years ago [6-9], and various laboratory investigations involving atomic clocks, see for example Refs. [10-13]. In contrast to studies involving analyses of the processes that have occurred billions of years ago, laboratory searches are sensitive to present-day variation of fundamental constants. Recently, our group's experiment utilizing the $E 1$ radio frequency (rf) transitions between nearly degenerate opposite parity levels in atomic dysprosium has yielded a result of $\dot{\alpha} / \alpha=(-2.7 \pm 2.6) \times 10^{-15} /$ yr 14 over an eight month observation period. A detailed description of the experimental setup and analysis was given in Refs. [14-16]. Here, we investigate a possible correlation between a change in $\alpha$ and a change in the gravitational potential.

The energy of an atomic level can be written as

$$
E=h \nu=E_{0}+q\left(\frac{\alpha^{2}}{\alpha_{0}^{2}}-1\right)
$$

where $E_{0}$ and $\alpha_{0}$ are the present-day values of the energy and $\alpha$, respectively, and $q$ contains the sensitivity of the level to $\alpha$ [17. From Eq. (1), a change in $\alpha$ should result in a change in $\nu$, for $\alpha \approx \alpha_{0}$, as given by

$$
h \delta \nu=2 q \frac{\delta \alpha}{\alpha} .
$$


Similarly, the change of the rf transition frequency between levels $A$ and $B$, as shown in Fig. 1, is given by

$$
\delta(\Delta \nu)=2 \frac{\left(q_{B}-q_{A}\right)}{h} \frac{\delta \alpha}{\alpha},
$$

where $\left|2\left(q_{B}-q_{A}\right) / h\right| \approx 1.8 \times 10^{15} \mathrm{~Hz}$ [17]. Here, we take this number to be exact, but the uncertainty is expected to be approximately twenty percent.

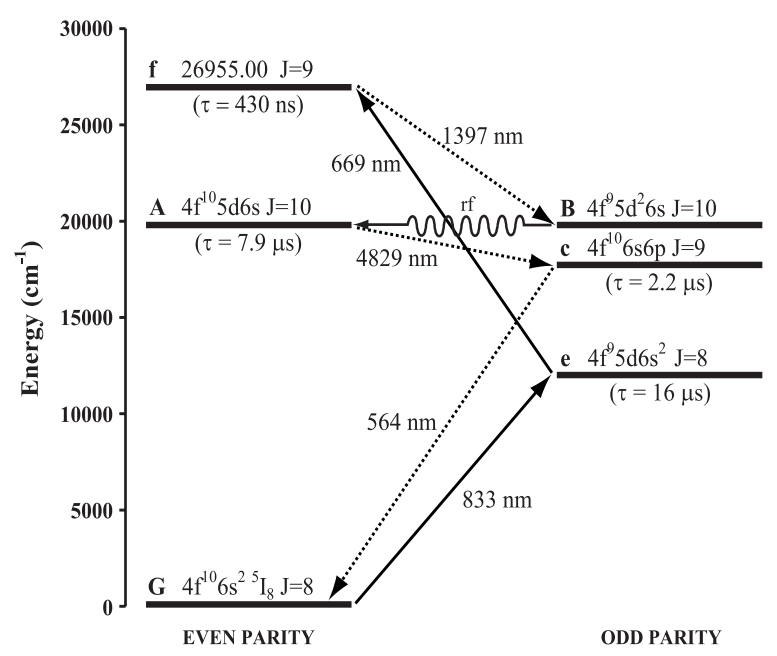

Figure 1: The relevant energy-level, population, and detection scheme for the dysprosium experiment.

The transitions utilized in the experiment are the $235-\mathrm{MHz}(\mathrm{J}=10 \rightarrow \mathrm{J}=10)$ transition of the isotope ${ }^{162} \mathrm{Dy}$ and the $3.1-\mathrm{MHz}(\mathrm{F}=10.5 \rightarrow \mathrm{F}=10.5)$ transition of ${ }^{163} \mathrm{Dy}$. The long-lived level $B$ (lifetime $\tau>200 \mu \mathrm{s}$ [18]) is populated via three transitions. Laser light at $833 \mathrm{~nm}$ and $669 \mathrm{~nm}$ is used for the first two transitions, and then the atoms spontaneously decay to level $B$ as shown in Fig. 1 The transition between levels $A$ and $B$ is induced by an applied rf electric field. The atoms decay from level $A$ in two steps, and the fluorescence at $564 \mathrm{~nm}$ is monitored to detect the rf transition.

Data were taken over an eight month period and hence at different points in the gravitational potential of the Sun. The gravitational potential at the Earth due to the Sun [19] is given by

$$
U=\frac{-G M_{s}}{r},
$$

where $G$ is the gravitational constant and $M_{s}$ is the mass of the Sun. For an elliptical orbit, the distance $r$ between the Sun and Earth is

$$
r=a \frac{1-\epsilon^{2}}{1+\epsilon \cos \phi},
$$

where $a$ is the semi-major axis of the Earth's orbit, $\epsilon=\sqrt{1-b^{2} / a^{2}} \approx 0.0167$ is the eccentricity, $b$ is the semi-minor axis, and $\phi$ is the true anomaly (see Fig. 2). Substituting Eq. (5) in to Eq. (4), the gravitational potential becomes

$$
U=\frac{-G M_{s}}{a}-\frac{G M_{s}}{a} \epsilon \cos \phi+O\left(\epsilon^{2}\right) .
$$

The first term in Eq. (6) is the constant part of the gravitational potential at Earth, while the second term is a change in gravitational potential, $\Delta U$, which arises due to the eccentricity of the Earth's orbit. The 

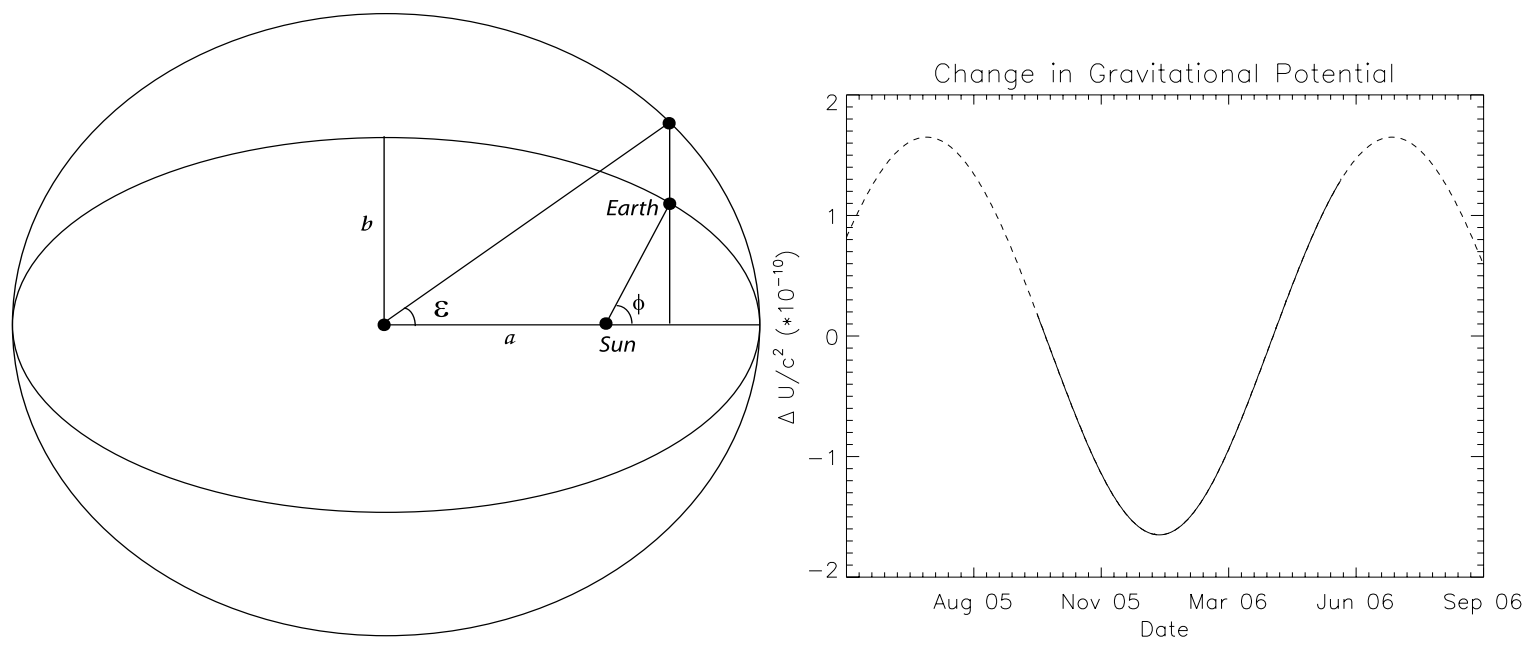

Figure 2: Left: The true anomaly, $\phi$, is the angle subtended from perihelion, the point of closest approach. The eccentric anomaly, $\varepsilon$, is the angle between perihelion and the position of the Earth in its orbit projected onto the auxiliary circle of the ellipse (the eccentricity of the ellipse is exaggerated for clarity). Right: The change in gravitational potential of the Sun at the Earth due to the ellipticity of the orbit. The solid line represents the time period during which data were taken.

fractional change in $\alpha$ as a function of gravitational potential can be parametrized as [20]

$$
\begin{aligned}
\frac{\delta \alpha}{\alpha} & =k_{\alpha} \frac{\Delta U(t)}{c^{2}}, \\
\Delta U(t) & =-\frac{G M_{s}}{a} \epsilon \cos \phi(t) .
\end{aligned}
$$

The data were taken between October 2005 to June 2006; the true anomaly is zero at the 2005 perihelion, January 2 [21. The true anomaly was calculated for each data point using a two step process. The elapsed time in days from perihelion is related to the eccentric anomaly (see Fig. 21) as [22]

$$
t=\sqrt{\frac{a^{3}}{G M}}(\varepsilon-\epsilon \sin \varepsilon),
$$

where $M=M_{s}+M_{E}$ is the sum of the masses of the Sun and Earth. The eccentric anomaly is obtained by solving Eq. (9) and then used to calculate the cosine of the true anomaly as a function of time since

$$
\cos \phi=\frac{\cos \varepsilon-\epsilon}{1-\epsilon \cos \varepsilon} .
$$

The calculated values for $\cos \phi$ are substituted into Eq. (8) to find the gravitational potential for each data point. The measured frequencies for each isotope are fitted to the gravitational potential using a two-parameter least squares fit given by

$$
\delta(\Delta \nu)-\nu^{*}=x_{0} \frac{\Delta U(t)}{c^{2}}+x_{1},
$$

where $\nu^{*}$ is an arbitrary reference frequency, and $x_{0}$ and $x_{1}$ are the fit parameters. The parameter $x_{1}$ accounts for the offset due to the reference frequency while the parameter $x_{0}$ determines the correlation between the change in gravitational potential and the transition frequency. If such a correlation is due to a change in $\alpha$, the sum and difference frequencies may also be fit to the varying potential to extract additional information 
regarding the variation of $\alpha$. Since the energy difference between levels $A$ and $B$ is of opposite sign for the two isotopes and the $q$ values are independent of the nucleus, the sum of the transition frequencies should be insensitive to $\alpha$ variation, while the difference of the transition frequencies should be twice as sensitive $[14,15]$. The data plots and the fits of the measured frequencies for each isotope as well as the sum and difference frequencies are shown in Fig. 3 .
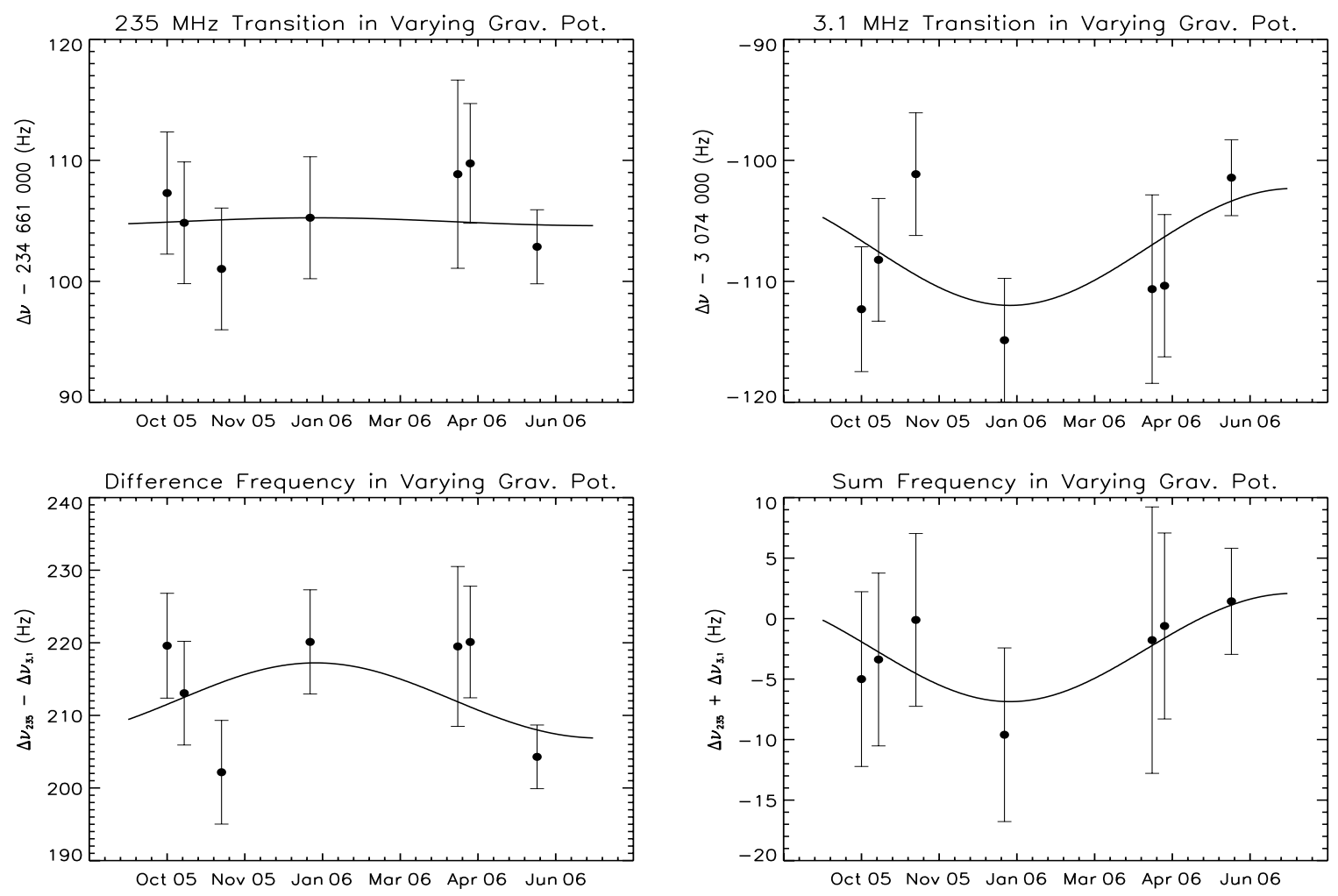

Figure 3: Top left: The data fitted to the gravitational potential for the 235-MHz transition. Top right: The data fitted to the gravitational potential for the 3.1-MHz transition. The bottom plots show the difference and sum frequencies fitted to the change in gravitational potential.

The parameter $x_{0}$ obtained by the least-squares fit is $(-0.2 \pm 1.7) \times 10^{10} \mathrm{~Hz}$ for the $235-\mathrm{MHz}$ transition, $(2.9 \pm 1.7) \times 10^{10} \mathrm{~Hz}$ for the $3.1-\mathrm{MHz}$ transition, $(2.7 \pm 2.4) \times 10^{10} \mathrm{~Hz}$ for the sum frequency, and $(-3.1 \pm 2.4) \times 10^{10} \mathrm{~Hz}$ for the difference frequency.

These values can be used to calculate the constraint on the parameter $k_{\alpha}$ from Eq. (7). Substituting Eq. (3) into Eq. (7), we get a relation similar to Eq. (11),

$$
\delta(\Delta \nu)=\left(2 \frac{q_{B}-q_{A}}{h}\right) k_{\alpha} \frac{\Delta U(t)}{c^{2}} .
$$

The fit parameter yields $k_{\alpha}=(-1.1 \pm 9.2) \times 10^{-6}$ for the $235-\mathrm{MHz}$ transition, $k_{\alpha}=(-16.3 \pm 9.4) \times 10^{-6}$ for the $3.1-\mathrm{MHz}$ transition, $k_{\alpha}=(-8.7 \pm 6.6) \times 10^{-6}$ for the difference frequency, and $(-7.5 \pm 6.6) \times 10^{-6}$ for the sum frequency. Although the value for the sum frequency is expected to be consistent with zero, the one-sigma mismatch of our value is consistent with the estimated uncertainties, dominated by systematic effects [14. 
The results relating a changing $\alpha$ to a changing gravitational potential are summarized in Table 1. The results in the first row of Table 1 depend on the variation of $\alpha$ as well as the variation of $m_{e} / m_{p}$. The dependence on $m_{e} / m_{p}$ is contained in $k_{e}$ [20. The results in the second and third rows in Table 1 depend on the variation of $\alpha$ and the variation of $m_{q} / m_{p}$, which is contained in $k_{q}$ [20. In addition to the results shown in Table 1 our results may limit the parameter range in some specific theories, such as those in Refs. [23-25].

Our calculated value of $k_{\alpha}$ can be combined with the results for $k_{\alpha}+0.17 k_{e}$ and $k_{\alpha}+0.13 k_{q}$ to extract a value of $(4.9 \pm 3.9) \times 10^{-5}$ for $k_{e}$ and $(6.6 \pm 5.2) \times 10^{-5}$ for $k_{q}$.

\begin{tabular}{c|c|c} 
Parameter & Constraint & Experimental Ref. \\
\hline \hline$k_{\alpha}+0.17 k_{e}$ & $(-3.5 \pm 6) \times 10^{-7}$ & {$[26]$} \\
\hline$\left|k_{\alpha}+0.13 k_{q}\right|$ & $<2.5 \times 10^{-5}$ & {$[27]$} \\
\hline$k_{\alpha}+0.13 k_{q}$ & $(-1 \pm 17) \times 10^{-7}$ & {$[28]$} \\
\hline$k_{\alpha}$ & $(-8.7 \pm 6.6) \times 10^{-6}$ & this work \\
\hline$k_{e}$ & $(4.9 \pm 3.9) \times 10^{-5}$ & this work \\
\hline$k_{q}$ & $(6.6 \pm 5.2) \times 10^{-5}$ & this work \\
\hline
\end{tabular}

Table 1: A summary of results for changing fundamental constants in a varying gravitational potential based on the theoretical work from Ref. 20.

In this paper, we have reported the first laboratory result for $k_{\alpha}$ and subsequently extracted a limit on $k_{e}$ and $k_{q}$. In principle the results from Ref. [26] can also be combined with other optical-Cs clock comparisons in such a way as to extract $k_{\alpha}$ independent of $k_{e}$. Currently, our results are limited by systematic uncertainties [14. A new apparatus is under construction to address the systematic effects and, thus, possibly increase the sensitivity of the experiment by up to three orders of magnitude [15.

The authors are grateful to M. Kozlov for stimulating discussions. This work has been supported in part by Los Alamos National Laboratory LDRD, NSF REU supplement, and by grant RFP1-06-15 from the Foundational Questions Institute (fqxi.org).

\section{References}

[1] J.-P. Uzan, Rev. Mod. Phys. 75, 403 (2003).

[2] J.K. Webb et al., Phys. Rev. Lett. 87, 091301 (2001).

[3] M.T. Murphy et al., Mon. not. R. Astron. Soc. 345, 609 (2003).

[4] R. Quast et al., Astron. Astrophys. 415, L7 (2004).

[5] R. Srianand, H. Chand, P. Petitjean, and B. Aracil, Phys. Rev. Lett. 92121302 (2004).

[6] T.Damour and F. Dyson, Nucl. Phys. B480, 37 (1996).

[7] Y. Fujii et al., Nucl. Phys. B573, 377 (2000).

[8] S.K. Lamoreaux and J.R. Torgerson, Phys. Rev. D 69, 121701(R) (2004).

[9] C.R. Gould, E.I. Sharapov, and S.K. Lamoreaux, Phys. Rev. C 74, 024607 (2006).

[10] S. Bize et al., Phys. Rev. Lett. 90, 150802 (2003).

[11] H. Marion et al., Phys. Rev. Lett. 90, 150801 (2003). 
[12] M. Fischer et al., Phys. Rev. Lett. 92230802 (2004).

[13] E. Peik et al., Phys. Rev. Lett. 93, 170801 (2004).

[14] A. Cingöz et al., Phys. Rev. Lett. 98, 040801 (2007).

[15] A.T. Nguyen, D. Budker, S.K. Lamoreaux, and J.R. Torgerson, Phys. Rev. A 69, 022105 (2004)

[16] A. Cingöz et al., Phys. Rev. A 72, 063409 (2005).

[17] V.A. Dzuba, V.V. Flambaum, and M.V. Marchenko, Phys. Rev. A 68, 022506 (2003).

[18] D. Budker, D. DeMille, E.D. Commins, and M.S. Zolotorev, Phys. Rev. A 50, 132-143 (1994).

[19] The variation of the gravitational potential due to Jupiter and the other planets was ignored since it is at least two orders of magnitude smaller than that due to the Sun.

[20] V.V. Flambaum, arXiv:0705.3704 physics.atom-ph, to be published in Int. J. Mod. Phys. A

[21] http://aa.usno.navy.mil/data/docs/EarthSeasons.html

[22] L. Hand and J. Finch, Analytical Mechanics, Cambridge University Press 1998.

[23] J. Magueijo, J.D. Barrow, and H.B. Sandvik, Phys. Lett. B 549, 284-289 (2002).

[24] H.B. Sandvik, J.D. Barrow, and J. Magueijo, Phys. Rev. Lett. 88, 031302 (2002).

[25] J. Magueijo,Phys. Rev. D 62, 103521 (2000).

[26] Fortier et al., Phys. Rev. Lett. 90, 070801 (2007).

[27] A. Bauch and S. Weyers, Phys. Rev. D 65, 081101R (2002).

[28] N. Ashby et al. Phys. Rev. Lett. 98, 070802 (2007). 